\title{
Advancing from gender equity to women in leadership in pediatric radiology
}

\author{
Cory M. Pfeifer ${ }^{1}$ (D) $\cdot$ Ami Gokli $^{2} \cdot$ Janet R. Reid ${ }^{2}$
}

Received: 15 October 2019 / Revised: 7 January 2020 / Accepted: 12 February 2020 / Published online: 10 April 2020

(C) Springer-Verlag GmbH Germany, part of Springer Nature 2020

The promotion of gender equity has emerged as a muchdiscussed topic in radiology. Women now represent the majority of medical school matriculants in the United States [1], but the proportion of women practicing radiology has remained relatively stable at approximately 25\% since 2012 [2]. Among radiologists, $30 \%$ of women and $10 \%$ of men work part-time [3], with women increasing in part-time preference at a slightly greater rate than men. Pediatric radiology, however, is the rare subset of diagnostic radiology in which there is a similar distribution of men and women, based on survey data [4] and a count of members with a public directory entry in the Society for Pediatric Radiology (SPR) online database [5]. In this paper we identify gender-related leadership trends in pediatric radiology while indicating areas of potential improvement. We also discuss strategies to meet the goal of increasing female presence in radiology and radiology leadership.

\section{Women's leadership in pediatric radiology}

The SPR was 38\% female in 2010 [6]. Women's membership in the Society of Chiefs of Radiology at Children's Hospitals (SCORCH) was reported to be $24 \%$ at that time. Since then, the proportion of women attending the annual SCORCH meeting has increased from 34\% in 2017 to $37 \%$ in 2018 and $41 \%$ in 2019. Despite this progressive interest in leadership among women, SCORCH presidents were all men from the genesis of the society in 1988 until 2018. In recent years, SCORCH has shifted toward a younger, more academically junior rank group.

Cory M. Pfeifer

Cory.Pfeifer@utsouthwestern.edu

1 Department of Radiology,

University of Texas Southwestern Medical Center,

5323 Harry Hines Blvd., Dallas, TX 75390, USA

2 Department of Radiology, Children's Hospital of Philadelphia, Philadelphia, PA, USA
More than $80 \%$ of SPR presidents have been men since the society's inception in 1958, though 58\% (7 of 12) of SPR presidents were men from 2006 to 2018. The SPR Board of Directors was $45 \%$ women in 2019. With an approximate 1:1 representation of women-to-men in SPR membership, these findings represent significant progress toward gender equity in SPR leadership, but there is still some opportunity for intervention in SCORCH.

The topic of unconscious bias has emerged in discussions related to gender discrepancies in physician leadership [7, 8]. Anti-bias training for practices that employ physicians has become perfunctory at many institutions but is not universal. The first step in preventing unconscious bias is realizing that it occurs. The SPR has an opportunity to identify and study the role of gender bias in pediatric radiology leadership and introduce interventions to move toward gender equity.

\section{Society for Pediatric Radiology Annual Meeting representation}

The SPR hosts an annual meeting that consists of a scientific session and an educational postgraduate course. Male and female participation in SPR-sponsored events was studied and presented at the 2019 meeting [5]. In 2018, 52\% of those who attended were women. For the postgraduate course, speakers are invited by the meeting organizers to deliver didactic lectures on the state of pediatric radiology imaging techniques; for the scientific sessions it is papers and not people that are chosen for presentation. In 2018, women made up $38 \%$ of the curriculum committee, $40 \%$ of the paper abstract review committee, and $53 \%$ of the poster abstract review committee. In the postgraduate course, $38 \%$ of the presenters were women, down from $40 \%$ in 2017 . Interestingly, $50 \%$ of the papers accepted for oral scientific presentation in 2018 were delivered by women, representing an increase from $46 \%$ in 2017. The fact that the selection of speakers for the postgraduate course depends on the organizers' recall of 
names of good speakers - a process that is not gender-blind - whereas the selection of papers is gender-blind does not go unnoticed.

It is plausible that the difference in gender representation at the 2018 SPR Annual Meeting between the postgraduate course and the scientific talks could be attributed to a slow "changing of the guard" because many scientific papers were presented by relatively junior radiologists, with equal representation of the sexes, compared to the invited speakers, who have more often been men than women. Nonetheless, such strong female representation in the number of registered attendees (52\%) and selected speakers for scientific sessions (50\%) suggests that women are interested in attending and speaking at this venue. Positive trends toward gender equity in the scientific sessions serve as food for thought in revising the selection process for the postgraduate course. A deliberate effort to groom and grow new female talent would serve the field well in moving closer to gender equity.

\section{Academic promotion and work-life balance}

Much literature has focused on academic promotion as both a measure of success and an indicator of gender bias, a metric germane to the $25 \%$ of the radiology workforce that is university-employed [2]. Academic radiology salaries have been suggested to be gender-neutral [9], with one study suggesting that radiology is the lone academic specialty in which women are paid slightly more than men [10]. In the case of pediatric radiology, approximately half of pediatric radiologists work in academically affiliated practices [11], but this descriptor includes radiologists employed by large private children's hospitals who have varying university affiliations. Pediatric radiology thus subsists as a mosaic subspecialty with a significant number of practicing radiologists who dwell in the gray area between private and academic practices. Academic promotion and salary discrepancies in pediatric radiology are thus difficult to evaluate. There is evidence to indicate that part-time pediatric radiology careers are associated with improved work-life balance [4], and the ability of radiologists to thrive as part-time clinicians is an achievable asset for the pediatric radiology subspecialty. Establishing a culture friendly to those interested in part-time clinical effort might be an effective strategy for other radiology subspecialties to attract women.

\section{Recommendations to women leaders}

\section{General}

The first step in attracting women leaders is to connect to our young female colleagues - to listen to women's stories and engender the belief that they have the potential to succeed. We need to develop a pipeline of female leadership. The many female role models in pediatric radiology might be operating on the sidelines and need to be given opportunities on the national stage. Mentoring and coaching programs are a natural mechanism to bring young talented women into the fold. Every effort must embrace the diverse expectations that women radiologists have, and this, in turn, might serve as a culture change and opportunity for men, as well. Now, more than ever, pediatric radiologists must celebrate the advances we have made in education and use these to create exciting career paths that distinguish us from our adult-focused colleagues. It is imperative that we pioneer methods to promote gender-blind selection of women to positions of training and leadership.

\section{Mentoring}

Promoting mentorship and sponsorship for women in radiology is a dominant theme in the literature. Mentorship can be defined along a spectrum from simply advising to coaching/ supporting. Sponsors, on the other hand, actively advocate for their colleagues and might put their own reputations at risk [12]. As one example developed to address the need for career growth, the Junior Society for Pediatric Radiology plans to add a speed-mentoring session to its annual meeting curriculum (M. Artunduaga, personal communication).

\section{Growing a pipeline through diverse practice options}

With its mix of available practice structures, pediatric radiology might be appealing to women who seek alternative career paths through education, mentoring and global partnerships, to name a few. Through a slow and deliberate practice that continues to identify and celebrate the needs of women in radiology, pediatric radiology can gradually attract women on the national stage as speakers, educators, scientists and leaders.

\section{Re-imagining leadership}

Representation in SPR-affiliated activities suggests that female pediatric radiologists want to lead and have their voices heard. Current SPR Annual Meeting content organizers have responded to this call for diversity by opening opportunities through limiting invited presentations to two per individual. The SPR and SCORCH must develop strategies to widen the lens of leadership to include women in its future. Diversity of representation will lead to even greater options in the future.

\section{Conclusion}

Pediatric radiology serves as an excellent laboratory to evaluate gender inclusion given its near-equivalent proportion of 
men and women. The landscape of leadership in pediatric radiology is slowly changing in response to this gender equilibration. By understanding factors that motivate pediatric radiologists, encouraging mentoring/sponsoring initiatives, and enacting policies that promote diversity, pediatric radiology is poised to serve as an example of gender inclusion for the entire field of radiology.

\section{Compliance with ethical standards}

Conflicts of interest None

\section{References}

1. Association of American Medical Colleges (2018) Matriculants to U.S. medical schools by sex, academic years 1980-1981 through 2018-2019. https://www.aamc.org/download/493012/data/ factsdatachart3.pdf. Accessed 1 Dec 2019

2. Bender CE, Bansal S, Wolfman D, Parikh JR (2019) 2018 ACR Commission on Human Resources workforce survey. J Am Coll Radiol 16:508-512

3. Bluth EI, Bansal S, Bender CE (2017) The 2017 ACR Commission on Human Resources workforce survey. J Am Coll Radiol 14: $1613-1619$
4. Menashe SJ, Parisi MT, Chapman T et al (2018) Part-time pediatric radiology: the realities and perceptions of part-time employment in the academic setting. AJR Am J Roentgenol 211:971-977

5. Gokli A, Pfeifer CM (2019) Gender representation in SPRsponsored events. Paper presented at the 62nd annual meeting \& postgraduate course of the Society for Pediatric Radiology. Pediatr Radiol 49:S137-S138

6. Boechat MI (2010) Women in pediatric radiology. Pediatr Radiol 40:484-487

7. Allen BJ, Garg K (2016) Diversity matters in academic radiology: acknowledging and addressing unconscious bias. J Am Coll Radiol 13:1426-1432

8. Abramson J, Fishman EK, Horton KM, Sheth S (2017) The cost of unconscious bias and pattern recognition. J Am Coll Radiol 14: $1119-1121$

9. Kapoor N, Blumenthal DM, Smith SE et al (2017) Sex differences in radiologist salary in U.S. public medical schools. AJR Am J Roentgenol 209:953-958

10. Jena AB, Olenski AR, Blumenthal DM (2016) Sex differences in physician salary in US public medical schools. JAMA Intern Med 176:1294-1304

11. Merewitz L, Sunshine JH (2006) A portrait of pediatric radiologists in the United States. AJR Am J Roentgenol 186:12-22

12. Deitte LA, McGinty GB, Canon CL et al (2019) Shifting from mentorship to sponsorship - a game changer! J Am Coll Radiol 16:498-500

Publisher's note Springer Nature remains neutral with regard to jurisdictional claims in published maps and institutional affiliations. 